\title{
Schulentwicklung an kleinen Schulen in ländlichen Regionen
}

\section{Bigna Sommer-Sutter}

Dieser Beitrag befasst sich mit Schulentwicklung an kleinen Schulen in ländlichen Regionen des alpinen Raums dreier Sprachregionen. Anhand von Fallbeispielen werden innovative lokale sowie regionale Lösungsansätze von kleinen Schulen diskutiert und es wird der Frage nachgegangen, welche Einflussgrössen innovatives Verhalten an kleinen Schulen fördern. Zusätzlich will der Beitrag Erfolgsfaktoren für die nachhaltige Implementation von Innovationen zur Erhaltung der kleinen Schulen in ländlichen Regionen herauskristallisieren.

\section{Einleitung}

«Nichts ist so beständig wie der Wandel»(Schumacher, 2008, S. 279).

Dieses Zitat von Schumacher charakterisiert das Wesen der Schulentwicklungsprozesse im Allgemeinen. Besonders betroffen vom Wandel sind aber kleine Schulen im alpinen Raum. Die Industrialisierung brachte räumliche Disparitäten bei Wirtschaft, Gesellschaft und Umwelt hervor. Städte wurden zu wichtigen wirtschaftlichen Zentren, ländliche Regionen und entlegene Standorte verloren an Bedeutung, was grosse Auswirkungen auf diese Gemeinden zur Folge hat ${ }^{1}$. Ab der zweiten Hälfte des 20. Jh. zeigen sich zunehmend kleinräumige Disparitäten: Gemeinden mit Bevölkerungswachstum und -rückgang innerhalb eines Tales oder einer Region. Siedlungswachstum erfasst höchstens die Hauptsiedlungen der grossen Transittäler, während die Weiler oder Streusiedlungen, die schlecht erreichbar sind, rückläufige Bevölkerungszahlen aufweisen (siehe dazu Michelet in dieser Nummer). Nebst den demographisch bedingten Voraussetzungen sind es auch bildungspolitische Forderungen, welche die Schulen vor neue Herausforderungen stellen und Schulentwicklungsprozesse auslösen. Je nach Region gibt es Unterschiede betreffend Bildungspolitik, während Vorarlberg sich in jeder Gemeinde für eine eigene Schule einsetzt, setzen die Kantone Graubünden, Wallis und St. Gallen auf deren Zusammenschlüsse. 
Schulentwicklungsprojekte von Einzelschulen häufen sich, wissenschaftlich erfasst werden sie kaum. Seit den 1990er Jahren werden Forschungsbeiträge verfasst, welche einzelne Teilbereiche der Schulentwicklung fokussieren wie z.B. Evaluation von Schulinnovationen, Analyse von Schulentwicklungsprojekten, Schulprogramm- und Steuergruppenarbeit. Eine umfassende Theorie der Schulentwicklung fehlt jedoch. Auch in ländlichen Regionen im alpinen Raum wächst die Zahl der Schulen, die Schulentwicklungsprojekte planen und durchführen (Kerle, 2007). Die vorliegende Studie, im Rahmen des grenzüberschreitenden Forschungsprojektes «Schule im alpinen Raum» der Pädagogischen Hochschulen Vorarlberg, Graubünden, St. Gallen und Wallis realisiert (Müller, Keller, Kerle, Raggl \& Steiner, 2011; Sutter \& Kerle, 2011), will hier anknüpfen und Schulentwicklungsprojekte an kleinen Schulen näher beleuchten und dokumentieren. Dieser Beitrag präsentiert zuerst vier innovative Fallbeispiele. In einem zweiten Schritt werden Einflussgrössen für Schulentwicklung in ländlichen Regionen beschrieben, und in einem dritten Schritt werden Erfolgsfaktoren für die Implementation von Schulentwicklungsprojekten an kleinen Schulen diskutiert.

Kleine Schulen (Kleinschulen) definiert die Interkantonale Arbeitsgemeinschaft externe Evaluation von Schulen (ARGEV) mit der Anzahl Abteilungen. Die kantonalen Fachstellen betrachten Schulen bis zu vier (einige bis zu sechs) Abteilungen als kleine Schulen (Hunziker, 2007). Als Abteilung wird die Klasse als geführte Einheit verstanden, welche eine oder mehrere Jahrgänge enthalten kann. Sie werden als soziale Bezugsgruppe gemeinsam unterrichtet. Das Projekt «Schule im alpinen Raum» hat Schuleinheiten mit weniger als 100 Schüler und Schülerinnen (an einer Primarschule) als «kleine Schule» und mit weniger als 50 als «sehr kleine Schule» definiert (Müller et al., 2011).

\section{Theoretische Einbettung}

\section{Schulentwicklung als Entwicklung von Einzelschulen}

Seit Ende der 1980er Jahre wird Schulentwicklung als ,Entwicklung der Einzelschule' verstanden (Rolff, 2006). Diese Sichtweise geht auf Forschungsergebnisse aus dem angelsächsischen Raum zurück, gemäss denen sich der Planungserfolg der Schule nicht auf der staatlichen Ebene, sondern auf der Ebene der Einzelschule entscheidet (Bohl, 2009; Rahm, 2005; Rolff, 2001, 2007). Fend (1986) führt das Konzept der ,einzelnen Schule als pädagogische Handlungseinheit' ein. Dalin und Rolff (1997) beschreiben in den 1990er Jahren die Einzelschulen als Motor der Schulentwicklung. Dalin (1999) sucht weiter nach Charakteristiken der Schulen als Organisation. Grunder (2002) versteht Schule als lernende Institution und beschreibt Schulentwicklung als «jene Lernprozesse, welche die betreffende Schule zu einem Umbau ihrer pädagogischen und institutionellen Struktur veranlassen» (S. 19). Dabei hebt er die Bedeutung des kollek- 
tiven Lernens hervor. Rolff (2007) geht weiter und versteht Schulentwicklung als Einheit zielgerichteter Gestaltung der Einzelschule im Systemzusammenhang von Unterrichts-, Organisations- und Personalentwicklung. Die Entwicklung einer Einzelschule ist nach Rolff mit der Entwicklung des gesamten Schulsystems verbunden, also auch mit Schulnetzwerken und Bildungsregionen.

Rolff (2007) betont, «dass ein Entwicklungsprozess nicht zu einem bestimmten Zeitpunkt beendet ist, sondern Schulen sich wieder aufs Neue veränderten Bedingungen anpassen müssen» (S. 17). Gemäss systemischem Ansatz entwickeln sich Schulen sowohl zufällig als auch geplant ständig weiter (Rolff, 2010). Dies ist besonders im alpinen Raum sehr deutlich zu spüren, da Schulen auf den demographischen Wandel antworten müssen.

Schulentwicklung kann demnach als stetiger Lernprozess verstanden werden. «Es geht dabei letztlich um die Einführung einer neuen Praxis durch Erfinden, Erproben oder Erneuern. Die Lernprozesse müssen vom Arbeitsplatz ausgehen und die Akteure dabei neue Einsichten gewinnen, ein anderes Verhalten zeigen, neue Wahrnehmungen machen, alte Routine aufgeben oder neue schaffen» (Rolff, 2007, S. 48).

Als Schulentwicklung wird auch die Implementation von Innovationen verstanden. Als Innovation wird eine neue pädagogische Idee, Methodologie, Verfahrensweise oder Technologie verstanden, welche Schulentwicklungen in Gang setzen kann (Schaumburg, Prasse \& Blömeke, 2010). Altrichter und Wiesinger (2005) verwenden den Begriff «Innovation» im Zusammenhang mit dem Begriff «Implementation», wobei die Autoren Implementation als Prozess der Überführung von Innovationen in Standardpraktiken beschreiben.

Die schulische Implementationsforschung beschäftigt sich mit der Frage, weshalb und unter welchen Bedingungen es gelingt, Innovationen nachhaltig in die Schulpraxis zu integrieren. Aus dem Vergleich der verschiedenen Implementationsmodelle (Warwas, Jürgen \& Meier, 2008) lässt sich schliessen, dass die Implementation von Innovationen in den Schulalltag in drei Phasen erfolgt, wobei diese Phasen in der Literatur bei gleichbleibendem Inhalt unterschiedlich bezeichnet werden: Die erste Phase ist die Planungsphase und dient der Analyse der Ausgangssituation und Zielfestlegung. Der Entwurf von Implementationsmassnahmen erfolgt in der zweiten Phase. Und die dritte Phase hat die Verfestigung der Strukturen, die Optimierung der implementierten Neuerungen und die Integration in den Arbeitsalltag zum Ziel.

Altrichter und Wiesinger (2005) zeigen auf, dass sich Implementation als schwieriger erweist, als es sich die Initianten von Schulinnovationen vorgestellt haben. Aus 18 untersuchten Fallbeispielen ziehen sie u.a. die Schlussfolgerungen, dass ein selektiver Modus die Übernahme von Neuerungen beeinflusst; es werden vor allem Elemente akzeptiert, welche mit der bestehenden Schulkultur leicht zu vereinbaren sind; und so schnell wie möglich realisiert werden können. 


\section{Schulentwicklung im spezifischen Kontext des alpinen} Raums

Der alpine Raum lässt sich nicht klar definieren. Jede Abgrenzung erfolgt von einem spezifischen Standpunkt aus und zu einem bestimmten Zweck. Gemäss Bätzings Standardwerk (2005) umfassen die Alpen einen Raum von 190’000 $\mathrm{km}^{2}$ in acht Staaten. 14 Millionen Menschen leben in 6124 Gemeinden, von denen $84 \%$ unter 1000 Höhenmetern liegen. Der alpine Raum in sich ist heterogen, durch Disparitäten geprägt, was zu Prozessen von Verstädterung bzw. Vervorstädterung und Entsiedlung führt (vgl. dazu Michelet in dieser Nummer sowie Michelet, Steiner, Périsset, Eyer \& Bumann, 2011). Diese Räume sind mit völlig unterschiedlichen Situationen, Problemen und Potenzialen konfrontiert. Verfügen die Agglomerationen über ein grosses kulturelles wie wirtschaftliches Angebot, sind die touristischen Destinationen durch den Saisonbetrieb gekennzeichnet (nur während der touristischen Saison verfügen die Destinationen über ein attraktives Angebot, in den Zwischensaisons werden sie zu ,Geisterstädten'). Nach Bätzing (2005) sind mögliche Alternativen zu dieser Entwicklung im alpinen Raum, die Lancierung von neuen Projekten und Initiativen, welche auf innovative Weise alpenspezifische Ressourcen nutzen und daraus neue Qualitätsprodukte entwickeln (in den Bereichen: Landwirtschaft, Waldnutzung, Energie, Gesundheit, Bauwesen, Handwerk, Kunsthandwerk, Tourismus, Gewerbe, Handel, Marketing, Architektur und wirtschaftliche Dienstleistungen). Diese Ansätze bedürfen einer hohen Innovationsbereitschaft. Dazu ist zu bedenken, dass ihre Protagonisten Menschen sind, denen es auf die unterschiedlichste Weise gelingt, traditionelle und moderne Werte produktiv $\mathrm{zu}$ verbinden, was der Schlüssel ihrer Innovationsfähigkeit ist. "Ohne solche kulturelle Innovationen sind wirtschaftliche Innovationen nicht vorstellbar» (S. 326).

\section{Studie im alpinen Raum: Fragestellung und Methode}

Die hier dargestellte Studie ist explorativ angelegt. Folgende Forschungsfragen stellen sich:

Wodurch zeichnen sich innovative lokale sowie regionale Lösungsansätze von kleinen Schulen in einem sich wandelnden ländlichen Raum aus?

Welche Einflussgrössen fördern bei kleinen Schulen innovatives Verhalten?

Gibt es Erfolgsfaktoren für die nachhaltige Implementation von Innovationen zur Erhaltung der kleinen Schulen in ländlichen Regionen?

Zur Beantwortung der Forschungsfragen wurden elf Primarschulen (insgesamt 80 Personen) aus drei Sprachregionen (Deutsch, Italienisch, Romanisch) mehrheitlich aus Graubünden, aber auch aus dem Wallis, aus St. Gallen und 
Vorarlberg - qualitativ mittels Interviewleitfaden erfasst. Zusätzlich wurde in den Kantonen Graubünden und Wallis sowie in Vorarlberg eine Fragebogenerhebung an 32 Schulen mit 130 Lehrpersonen und 45 Schulräten durchgeführt. Die elf Schulen, welche als Fallbeispiele in der qualitativen Untersuchung aufgearbeitet wurden, sind Schulen, die explizit ein Schulentwicklungsprojekt durchgeführt und Neuerungen implementiert haben bzw. kurz vor der Implementation derselben stehen. Für diesen Beitrag wurden vier erfolgreiche Projekte (innovatives ,Nischenprodukt' in der Region) ausgewählt und folgend als Fallbeispiele dargestellt.

\begin{tabular}{|c|c|c|c|c|}
\hline Schulen & $\begin{array}{l}\text { Anzahl Schüler } \\
\text { und Schülerinnen }\end{array}$ & $\begin{array}{l}\text { Schulstufen } \\
\text { 2008-2009 }\end{array}$ & Schulsprachen & Anzahl Interviews \\
\hline A & 29 & 1.-3. / 4.-6. & $\mathrm{R} / \mathrm{D}$ & 8 \\
\hline B & 60 & 1.-2. / 3.-4. / 5.-6. & I & 7 \\
\hline $\mathrm{C}$ & 17 & 1.-3 / 4.-6. & $\mathrm{I} / \mathrm{D}$ & 9 \\
\hline $\mathrm{D}$ & 24 & 1.-6. & $\mathrm{D}$ & 6 \\
\hline $\mathrm{E}$ & 55 & 1.-2./3.-4. / 5.-6. & $\mathrm{R}$ & 8 \\
\hline $\mathrm{F}$ & 82 & $\begin{array}{l}1 . / 2 . / 3 . / 4 . / 5 . \\
/ 6 .\end{array}$ & $\mathrm{R} / \mathrm{D}$ & 7 \\
\hline G & 43 & 1.-2. /3.-4. / 5.-6. & $\mathrm{R}$ & 8 \\
\hline $\mathrm{H}$ & 66 & 1.-2. /3.-4. / 5.-6. & $\mathrm{D}$ & 5 \\
\hline I & 46 & 1.-2./3.-4. / 5.-6. & $\mathrm{D}$ & 5 \\
\hline K & 50 & $1 . / 2 . / 3 . / 4$. & $\mathrm{D}$ & 5 \\
\hline $\mathrm{L}$ & 26 & $1 .-3 / 4 .-6$ & $\mathrm{D}$ & 6 \\
\hline Inspektoren & & & & 6 \\
\hline
\end{tabular}

$\mathrm{R}=$ Rätoromanisch $/ \mathrm{D}=$ Deutsch $/ \mathrm{I}=$ Italienisch

Pro Schulträgerschaft wurden fünf bis neun Interviews geführt. Der Kreis der Befragten wurde so ausgewählt, dass aus allen Akteurgruppen eine bis drei Personen vertreten waren, damit die Schule und deren Entwicklung aus verschiedenen Blickwinkeln beleuchtet werden konnte. Folgende Personengruppen wurden in der Untersuchung berücksichtigt: Kantonale Aufsichtsbehörden (Inspektoren), Gemeindebehörden, Entscheidungsträger (lokale Schulaufsichtsbehörde: Schulratsmitglieder, meist von der Gemeindeversammlung gewählt), Schulleitungen, Lehrpersonen, Eltern sowie Schülerinnen und Schüler. Unter den untersuchten Schulen in den vier Projektregionen finden sich deutschsprachige Schulen (Mehrheit), rätoromanisch- oder italienischsprachige Schulen sowie zweisprachige Schulen (deutsch-rätoromanisch und deutsch-italienisch).

Die genannten Akteure einer Schule wurden betreffend Planung und Durchführung ihrer konkreten Schulentwicklungsprojekte an ihrer Schule vor Ort befragt. Der eigens für diese Arbeit konstruierte Leitfaden basiert 
teilweise auf dem Interviewleitfaden, welcher schon für die Fallstudie von Kerle (2007) verwendet wurde. Er umfasst die Themenbereiche Motivation, auslösende Faktoren und Initiatoren von Schulentwicklungsprojekten sowie den Verlauf des Schulentwicklungsprozesses auf der Grundlage der eingeführten Massnahmen sowie die Schul- und Regionalentwicklung. Die Auswertung der Interviews erfolgte in einer ersten Phase mittels qualitativer Inhaltsanalyse nach Mayring (2008); anschliessend wurde mittels thematischem Ansatz nach Flick (2000) eine weitere Auswertung vorgenommen, welche den Vergleich der Schulentwicklungsprozesse der untersuchten Schulen ermöglicht.

In der flächendeckenden Befragung mittels Fragebogen ( $\mathrm{n}=130$ Lehrpersonen / 45 Schulräte) wurden die Skalen ,Mitspracherecht der Lehrpersonen' sowie ,Innovationsbereitschaft' eruiert. Die zwei Skalen wurden faktoranalytisch überprüft. Die gewonnenen Faktoren wurden mittels Varimax-Methode rotiert und im Hinblick auf Reliabilitätsanforderungen nach dem Konsistenzkoeffizienten (Cronbachs-Alpha) untersucht. Die Skala ,Mitspracherecht der Lehrpersonen' weist einen Cronbach-Alpha von .80 auf, sie verteilt sich angehend symmetrisch mit einem $M$ von 22.21 und einer $S D$ von 4.19. Die Skala, Innovationsbereitschaft' weist einen Cronbach-Alpha von .64 auf, auch sie verteilt sich angehend symmetrisch mit einem $M$ von 12.07 und einer $S D$ von 2.04.

\section{Ergebnisse}

Die Ergebnisse der alpinen Schulentwicklungsstudie werden in drei Schritten dargestellt: Zuerst werden Fallbeispiele von vier erfolgreichen innovativen Primarschulen (darunter eine Grundschule) mit ihren Lösungsansätzen (was eingeführt wurde, weshalb, personelle Änderungen etc.) abgebildet. In einem zweiten Schritt werden Einflussgrössen für die Schulentwicklung an kleinen Schulen vorgestellt. Und schliesslich werden Erfolgsfaktoren für die Implementation von Schulentwicklungsprojekten an kleinen Schulen diskutiert.

\section{Vier innovative Primarschulen}

Aus den Interviews ist zu entnehmen, dass sich innovative Schulen durch systematische Schulentwicklung auszeichnen. Diese muss im Einklang mit den Bedürfnissen der Region und Hand in Hand mit dieser geplant und eingeführt werden. Altersdurchmischte Lerngruppen stellen eine mögliche innovative Lösung zur Erhaltung einer kleinen Schule in ländlichen Ortschaften dar. Im Folgenden werden vier Schulen, welche sich durch die Ausarbeitung und Umsetzung eines innovativen Konzeptes von den anderen abheben, portraitiert. Die Ortschaft liegt auf einem Pass, der zugleich die Sprachgrenze italienisch-deutsch/romanisch bildet. Politisch gehört die Ortschaft zur Talgemeinde, die italienischsprachig ist. 16 Kilometer entfernt liegt eine wichtige touristische Ortschaft. Im Jahre 2002 fiel der Entscheid, eine zweisprachige Schule einzurichten. 


\section{Schule C: Einführung einer zweisprachigen Schule (italienisch-deutsch)}

\begin{tabular}{|l|l|}
\hline \multicolumn{2}{|l|}{ Schulorganisation der Schule C } \\
\hline Einzugsgebiet & $\begin{array}{l}\text { Ortschaft mit zwei Fraktionen (570 Ew.), liegt } \\
\text { an einem Pass, politisch eine Talgemeinde } \\
1815 \text { m.ü.M. }\end{array}$ \\
\hline Schulbehörde & drei Mitglieder \\
\hline Schulleitung & Seit 2009 für das ganze Tal \\
\hline Schulangebote & Freiwilligen-Mittagstisch \\
\hline & $\begin{array}{l}17 \\
\text { zwei Abteilungen: } \\
\text { (Schuljl Schüler/-innen (1.-6. Kl.) }\end{array}$ \\
& $\begin{array}{l}1 .-3 . \text { Kl. }=7 \\
\text { 4. -6. Kl. }=10\end{array}$ \\
\hline
\end{tabular}

Das Jahr 2003 wurde zur Projektvorbereitung genutzt. Seit dem Schuljahr 2004-2005 wird die Schule zweisprachig geführt. Auf das Schuljahr 2009-2010 hin wurde zudem eine Schulleitung für alle Schulen im Tal eingesetzt, da die drei Gemeinden zu einer Schulgemeinde fusionierten.

Die Schülerinnen und Schüler besuchen nun bereits ab dem Kindergarten einen immersiven Unterricht, in welchem die beiden Sprachen zu je 50\% als Unterrichtssprache eingesetzt werden. Konkret wird der Unterricht wie folgt gestaltet: Da die Kindergartenlehrperson zweisprachig ist, spricht sie je nach Tageszeit und Unterrichtsprogramm Deutsch oder Italienisch.

In der Primarschule unterrichten zwei Lehrpersonen (Schuljahr 2009-2010) insgesamt 17 Schülerinnen und Schüler, die in zwei Abteilungen aufgeteilt sind (1.-3. und 4.-6. Klasse). Eine Lehrperson ist für die Fächer zuständig, die in Deutsch unterrichtet werden, da ihre Muttersprache Deutsch ist. Die zweite Lehrperson ist für diejenigen Fächer zuständig, die in Italienisch erteilt werden, da ihre Muttersprache Italienisch ist. Die Fächer Mathematik, Geografie und Geometrie werden in Deutsch unterrichtet (dies ist von den kantonalen Behörden so vorgegeben), die Fachbereiche Geschichte sowie Mensch und Umwelt in Italienisch. Die anderen Fächer werden je nach Vorlieben und Fähigkeiten der zwei Lehrpersonen aufgeteilt, wobei die 1.-3. Klasse mehr Fächer in deutscher Sprache, die 4.-6. Klasse ein wenig mehr in italienischer Sprache besucht. Auf die sechs Schuljahre verteilt gleicht sich dies aber aus.

Eine Lehrperson berichtet von den Vorteilen und den Schwierigkeiten, die mit dem immersiven Unterricht verbunden sind. Sie ist der Meinung, dass die Schülerinnen und Schüler in einer zweisprachigen Schule viel flexibler denken lernen. Ein «Hin- und Herwechseln» zwischen den beiden Sprachen bereitet den Schülerinnen und Schülern keine Mühe. Eine Herausforderung stellen jedoch fehlende didaktische Materialien dar, welche für eine zweisprachige Schule konzipiert sein sollten.

Für die Einführung der zweisprachigen Schule wurde eine deutschsprachige Lehrperson eingestellt, welche die italienische Sprache mündlich und schrif- 
tlich gut beherrscht. Auch im Kindergarten kam es zu einem Personalwechsel, der jedoch nicht mit dem Schulentwicklungsprojekt in Verbindung stand. Es wurde neu eine zweisprachige Lehrperson eingestellt. Das Lehrerteam konnte an einzelnen Tagen an anderen zweisprachigen Schulen, u.a. auch an Schulen in Italien, hospitieren und hat sich speziell zur Thematik des zweisprachigen Unterrichtens weitergebildet. Die Schule wird von der Pädagogischen Hochschule Graubünden im Auftrag des AVS (Amt für Volksschule und Sport Graubünden) als Schulprojekt begleitet und auf ihre Wirksamkeit hin untersucht.

\section{Schule E: Einführung einer Tagesschule}

\begin{tabular}{|l|l|}
\hline Schulorganisation der Schule E \\
\hline Einzugsgebiet & $\begin{array}{l}\text { Gemeindegebiet (300 Ew.) } \\
166^{2} \text { m.ü.M. }\end{array}$ \\
\hline Schulbehörde & fünf Mitglieder \\
\hline Schulleitung & Keine \\
\hline Schulangebote & Tagesschule von 7.45-18.00 Uhr (Montag- \\
& Freitag, am Mittwoch von 7.45-12.00 Uhr) \\
\hline & 55 \\
Anzahl Schüler/innen (1.-6. Kl.) & Drei Abteilungen: \\
(Schuljahr 2009-2010) & $\begin{array}{l}\text { 3.-2. Kl. }=22 \\
\end{array}$ \\
& 5.-6. Kl. $=13$ \\
& \\
\hline
\end{tabular}

Im Jahre 2006 wurde die Einführung einer Tagesschule im Gemeinderat besprochen. Auf das Schuljahr 2006-2007 hin konnte die Tagesschulstruktur eingeführt werden. Bereits vor der Reorganisation hatte eine Lehrperson Hausaufgabenhilfe für die eigene Klasse angeboten. Mit der Reorganisation wurde dieses Angebot nun für alle Klassen zugänglich gemacht. Dank der Bereitschaft der Lehrpersonen, die Hausaufgabenhilfe und den Mittagstisch (im Restaurant oberhalb der Schule) zu übernehmen und somit mehr als vor der Reorganisation $\mathrm{zu}$ arbeiten, konnte die Umstrukturierung vollzogen werden. Heute arbeiten die Lehrpersonen intensiver zusammen als vor der Reorganisation, da sie zusätzlich die Hausaufgabenhilfe und den Mittagstisch gemeinsam zu planen und zu koordinieren haben.

Die Tagesschule bietet Blockzeiten, einen Mittagstisch und Hausaufgabenhilfe an. Die Familien können ihre Kinder für den Mittagstisch sowie für die Hausaufgabenhilfe, die am Morgen vor Schulbeginn, am Mittag und am Nachmittag nach Schulschluss angeboten wird, anmelden. Eine Stunde Hausaufgabenhilfe pro Tag kann von den Kindern kostenlos in Anspruch genommen werden. Weitere betreute Stunden sowie die Teilnahme am Mittagstisch sind kostenpflichtig.

Aufgrund der Pensionierung der bisherigen Lehrperson der 1./2. Klasse wurde eine neue Lehrperson eingestellt. Bereits im Vorstellungsgespräch wurde deren 
Bereitschaft, im System der Tagesschule zu arbeiten, geklärt. Die Lehrpersonen vertreten den Standpunkt, dass eine solche Schule nur funktionieren kann, wenn alle Lehrpersonen sich dafür engagieren. Gefragt ist nicht nur die Beteiligung der Hauptlehrpersonen, sondern auch diejenige der Teilzeitlehrpersonen. Auch eine Stellvertretungsregelung betreffend Mittagstisch- und Hausaufgabenhilfebetreuung wurde in Absprache mit den Lehrpersonen festgelegt. Obwohl von den Lehrpersonen neu mehr Stunden für denselben Lohn geleistet werden, zeigen sich diese mit der getroffenen Lösung zufrieden.

\section{Schule F: Einführung einer zweisprachigen Schule (deutsch-rätoromanisch)}

\begin{tabular}{|l|l|}
\hline \multicolumn{2}{|l|}{ Schulorganisation der Schule F } \\
\hline Einzugsgebiet & Gemeindegebiet, bestehend aus drei \\
& Fraktionen (1208 Ew.) \\
& Ortschaft am Taleingang \\
& 875 m.ü.M. \\
\hline Schulbehörde & fünf Mitglieder \\
\hline Schulleitung & im Teilpensum \\
\hline Anzahl Schüler/innen (1.-6. Kl.) & 82 \\
(Schuljahr 2009-2010) & Je nach Kinderzahlen fünf od. sechs Abtei- \\
& lungen: \\
& 1. Kl. $=15$ \\
& 2. Kl. $=19$ \\
& 3. Kl. $=11$ \\
& 4. Kl. $=11$ \\
& 5. Kl. $=12$ \\
& 6. Kl. $=14$ \\
\hline
\end{tabular}

Der Unterricht in Rätoromanisch war 2001-2002 Anlass für eine rege Diskussion an der Schule, denn die hier beschriebene Gemeinde liegt geografisch gesehen zwischen den Sprachgebieten Deutsch und Rätoromanisch, 15 Kilometer von Chur entfernt. Die Amtssprache der Gemeinde ist Rätoromanisch, demnach galt Rätoromanisch vor der Reorganisation als Schulsprache. Lediglich ein Drittel der Kinder sprechen zu Hause Rätoromanisch. Einige Eltern setzten sich dafür ein, Deutsch als Schulsprache festzusetzen. Dies führte dazu, dass auf das Schuljahr 2003-2004 hin das Pilotprojekt einer zweisprachigen Schule lanciert wurde. In der Folge beteiligte sich die Schule ab dem Schuljahr 2006-2007 am Schulprojekt Rumantsch Grischun, das durch den Kanton Graubünden initiiert wurde. Das Projekt sieht vor, den Unterricht in Rumantsch Grischun, der offiziellen rätoromanischen Amtssprache, und nicht mehr in dem ortsüblichen rätoromanischen Idiom zu halten. Das Projekt Rumantsch Grischun wird heute immer noch von der Pädagogischen Hochschule Graubünden im Auftrag des Amt für Volksschule und Sport des Kantons Graubünden (AVS) begleitet, mit dem Ziel, die Wirksamkeit und die Praxistauglichkeit in den Schulen zu prüfen.

An der Schule F wird der Unterricht seit der Reorganisation zu je $50 \%$ in Deutsch und 50\% in Rätoromanisch gehalten. Je nach Schulfach wird Deutsch 
oder Rätoromanisch als Unterrichtssprache verwendet. Mathematik erfolgt in deutscher Sprache, die anderen Hauptfächer je zur Hälfte in deutscher und in rätoromanischer Sprache, der Unterricht in den Nebenfächern wie Zeichnen, Singen und Sport in Rätoromanisch. Im Kindergarten spricht die Lehrperson beide Sprachen, wobei die Mehrheit der Kinder eher in Deutsch antwortet, da sie deutscher Muttersprache sind. In der Schule hängt im Klassenzimmer jeweils eine Tafel, die anzeigt, welche Sprache aktuell gesprochen werden sollte. Auf dem Pausenplatz wird jedoch vorwiegend Deutsch kommuniziert.

Die Lehrpersonen, die an der Schule arbeiten, sind alle rätoromanischer Muttersprache und sprechen gut Deutsch. Es werden nur Lehrpersonen eingestellt, welche beide Sprachen mündlich wie auch schriftlich beherrschen. Das Konzept der Schule wurde von einer anderen zweisprachigen Schule übernommen und an die eigenen Bedürfnisse (z.B. Fach Mathematik in deutscher Sprache) angepasst. Das Institut für Mehrsprachigkeit der Universität Fribourg bildete die Lehrpersonen weiter, wobei v.a. die Vorteile des zweisprachigen Unterrichts aufgezeigt und für den Unterricht nutzbar gemacht wurden. Zusätzlich wurden weitere Weiterbildungsangebote, unter anderen auch solche, die von der Pädagogischen Hochschule Graubünden durchgeführt wurden, von den Lehrpersonen besucht, um sich auf die veränderte Schulsituation vorzubereiten. Unterstützt wurde die Einführung des zweisprachigen Unterrichts durch eine Lehrmitteleinführung für Rumantsch Grischun, die durch das Amt für Volksschule und Sport des Kantons Graubünden (AVS) initiiert wurde.

\section{Schule K: Einführung des altersgemischten Unterrichts}

\begin{tabular}{|l|l|}
\hline \multicolumn{2}{|l|}{ Schulorganisation der Schule K } \\
\hline Einzugsgebiet & $\begin{array}{l}\text { Gemeindegebiet (1012 Ew.) } \\
861 \text { m.ü.M. }\end{array}$ \\
\hline Schulbehörde & Keine Gemeindeschulbehörde (sondern \\
& Bezirksschulinspektor, Landesschulinspektor \\
& und Sonderschulinspektor) \\
\hline Schulleitung & im Teilpensum \\
\hline Anzahl Schüler/innen (1.-4. Kl.) & 50 \\
(Schuljahr 2009-2010) & vier Abteilungen, je nach Bedarf drei Abtei- \\
& lungen: \\
& 1. Kl. $=11$ \\
& 2. Kl. $=19$ \\
& 3. Kl. $=15$ \\
& 4. Kl. $=5$ \\
\hline
\end{tabular}

Seit dem Schuljahr 2006-2007 ist die Grundschule (1.-4. Klasse) dreiklassig. Es wird altersgemischt unterrichtet, je nach Situation die 1. und die 2. Klasse oder die 3. und die 4. Klasse zusammen. Auf das Schuljahr 2009-2010 wurde ein neues Modell des altersgemischten Unterrichts eingeführt, in dem die 1. und 4. Klasse gemeinsam unterrichtet werden, manchmal auch die 3. und 
4. Klasse. Die 1. und die 4. Klasse werden gemeinsam unterrichtet, wobei die 4. Klasse am Wochen- bzw. Tagesplan arbeitet. Die Lehrperson unterrichtet je nach Thematik und Situation die Erstklässler oder die Viertklässler auch alleine. Der Sachunterricht und der Musikunterricht werden immer gemeinsam durchgeführt. Darüber hinaus wurde ein schulstufenübergreifendes Förderkonzept entwickelt, in welches auch der Kindergarten integriert ist. Gemeinsame Projekte des Kindergartens und der Grundschule werden als Möglichkeit angesehen, die Sozialkompetenzen zu stärken und Kindergartenkinder mit dem Schulbetrieb vertraut zu machen. So lesen beispielsweise die Schulkinder den Kindergartenkindern im Projekt «Lesefreunde» vor. Die Kindergartenkinder kommen dadurch auch in die Bibliothek, beteiligen sich an vorschulischen Programmen und werden dabei von den Schulkindern unterstützt.

Der Einsatz der Lehrpersonen wird jedes Jahr neu organisiert, wobei die aktuellen Bedürfnisse der Kinder und der Lehrpersonen Berücksichtigung finden. Die Lehrpersonen sprechen sich $\mathrm{ab}$, wer wofür am besten geeignet ist. Bereits vor der Reorganisation unterrichteten sie im Teamteaching und konnten so besser auf den aktuellen Lernstand der Kinder eingehen. Aufgrund der Umstellung auf altersgemischten Unterricht besuchen die Lehrpersonen auch Weiterbildungsveranstaltungen zum Thema Kleinschule. In der Region existiert zudem eine Gruppe, welche sich speziell mit der Thematik Kleinschule auseinandersetzt und ein grosses Engagement dafür zeigt.

\section{Einflussgrössen, welche innovatives Verhalten an kleinen Schulen fördern}

Die treibenden Faktoren für Entwicklungsvorhaben an kleinen Schulen sind vor allem im Bereich der Rahmenbedingungen zu suchen, mit denen sich die Schulen konfrontiert sehen:

- Niedrige Schülerzahlen

- Wunsch, die Schule im Dorf zu behalten

- Finanzielle Faktoren

- Angst vor Fusionen mit anderen Schulen

- Druck von Seite des Kantons

- Sprachensituation in der Gemeinde

- Steigerung der Attraktivität der Schule

- Steigerung der pädagogischen Qualität.

Von besonderer Bedeutung ist das Motiv, die Schule oder wenigstens Teile davon trotz sinkender Schülerzahlen im eigenen Dorf zu erhalten. Für dieses Ziel ist die Bereitschaft vorhanden, Veränderungen in Kauf zu nehmen. Oft wird versucht, gleichzeitig mit der angestrebten Innovation auch die Attraktivität der Schule zu steigern, damit wieder mehr Familien mit Kindern in die Region bzw. ins Dorf ziehen.

Es zeigt sich, dass Unterschiede bezüglich auslösender Momente zwischen 
den untersuchten Schulen bestehen. So sind nebst Top-down- auch Bottom-upProzesse zu verzeichnen. $\mathrm{Zu}$ bemerken ist, dass die im vorangehenden Abschnitt als innovativ bezeichneten Schulen von der Basis her (Bottom up) entwickelt wurden. Die treibenden Kräfte der Entwicklungsprozesse sind speziell für das Entwicklungsvorhaben eingesetzte Arbeitsgruppen oder auch häufig Einzelpersonen. Dem Leader kommt im Prozess eine grosse Bedeutung zu, denn ohne ihn würden solche Prozesse oft nicht zu Ende geführt werden. Welche Person diese Funktion übernimmt, variiert von Projektregion zu Projektregion, da die Schulsysteme unterschiedlich aufgebaut $\operatorname{sind}^{2}$ und unterschiedlich gesteuert werden.

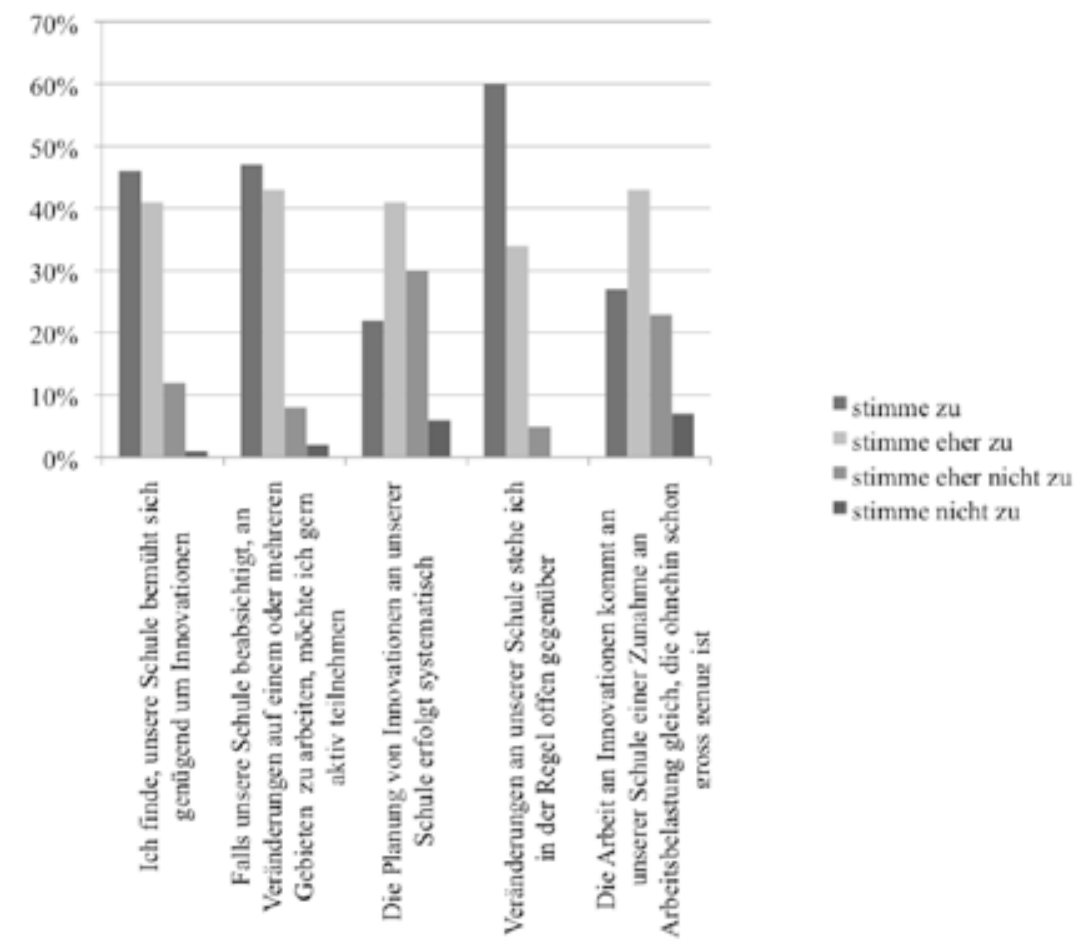

Abbildung 1: Einstellung von Lehrpersonen zu Veränderungsprozessen $(n=130)$

Oft wird die Funktion des Leaders vom Schulratspräsidenten, der Schulleitung oder von einer engagierten Lehrperson übernommen. Es zeigt sich, dass vor allem in Graubünden und im Wallis der Funktion des Schulrates, im Speziellen derjenigen des Schulratspräsidenten, eine besondere Rolle zukommt. In Schulen, in denen die Schulleitung nicht vorhanden ist, übernehmen die Schulratspräsidenten häufig deren Funktion. 
Zusätzlich zeigt sich aus der Analyse des Fragebogens, dass Lehrpersonen an kleinen Schulen sehr innovationsbereit und offen gegenüber Veränderungsprozessen sind. Dies kann als Einflussfaktor (gleichzeitig jedoch auch als Erfolgsfaktor) für Schulentwicklung angesehen werden.

In der Abb. 1 sieht man, dass Primarlehrpersonen (aus Vorarlberg, Graubünden und Wallis) Veränderungsprozessen an ihrer Schule eher offen gegenüber stehen (94\% stimmen zu bzw. stimmen eher zu). Im Weiteren stimmen sie der Aussage zu bzw. eher zu, dass sich ihre Schule genügend um Erneuerungen oder Innovationen bemüht (87\%). Der Aussage, Die Lehrpersonen beabsichtigen aktiv an Veränderungen der eigenen Schule teilzunehmen' stimmen 90\% zu bzw. eher zu. Es zeigt sich jedoch auch, dass die Veränderungen an den Schulen nicht systematisch erfolgen.

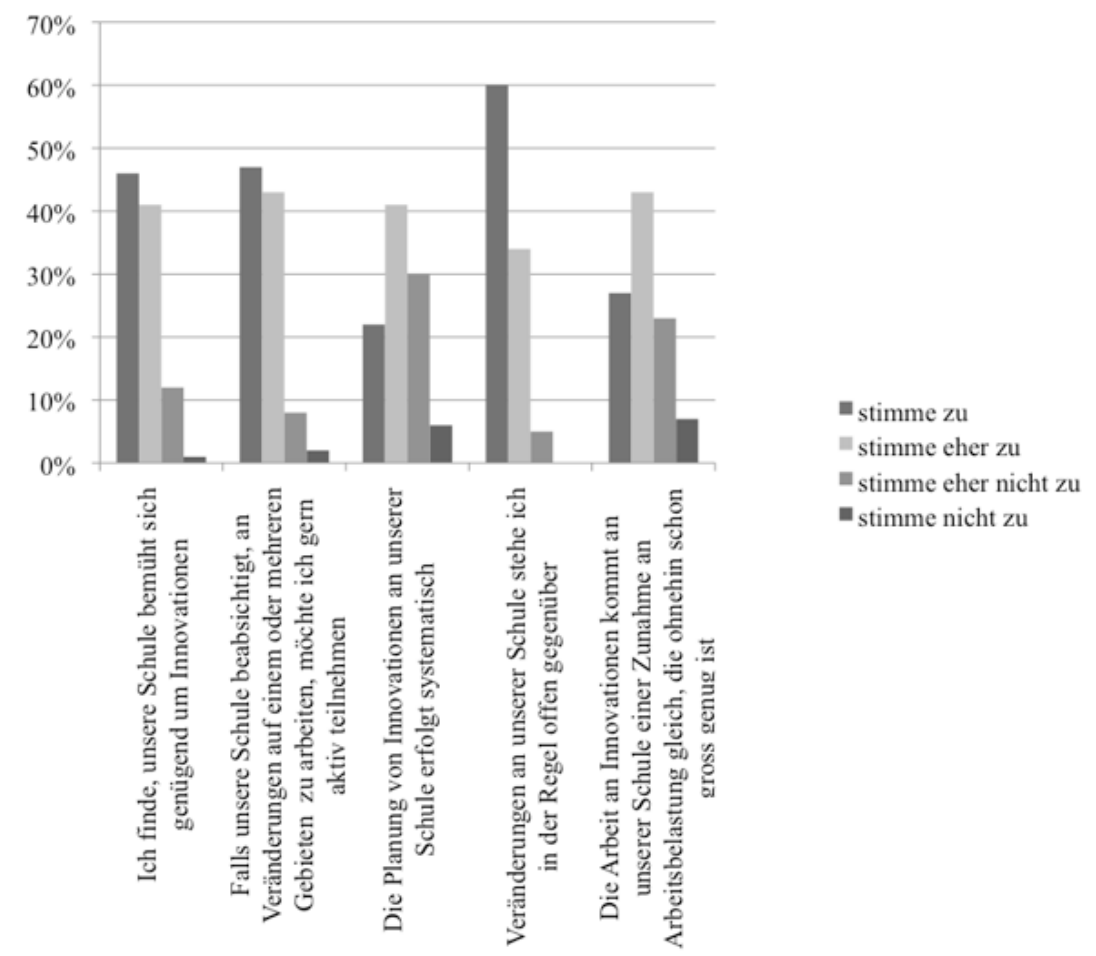

Abbildung 2: Mitspracherecht der Lehrpersonen $(n=130)$

Abb. 2 zeigt, dass die Lehrpersonen an den kleinen Schulen in einigen Bereichen viel Mitspracherecht haben. Beispielsweise beim ,Stundenplan' (64\%),Verteilung der Aufgaben unter den Lehrpersonen' (56\%) und ,Wahl von Lehrmitteln' (61\%). In sieben von zehn Bereichen geben mehr als 80\% der befragten Lehrpersonen an, ,viel Mitspracherecht' oder, ein wenig Mitspracherecht' zu haben. 
Zusätzlich ist aus den Analysen zu entnehmen, dass zwischen den Skalen «Mitspracherecht der Lehrpersonen» und "Innovationsbereitschaft» ein signifikanter positiver linearer Zusammenhang $(r=.42, p<.01)$ besteht. Je mehr Mitspracherecht Lehrpersonen an einer kleinen Schule zu haben empfinden, desto höhere Werte weisen sie auch auf der Skala der «Innovationsbereitschaft» auf und umgekehrt.

\section{Erfolgsfaktoren für die nachhaltige Implementation von Innovationen zur Erhaltung der kleinen Schulen in ländlichen Regionen}

Aus den qualitativen Daten ergibt sich, dass die Erfolgsfaktoren, die aus den bisherigen Erkenntnissen aus der Schulentwicklungsforschung (Rolff, 2007) gewonnen wurden, auch für kleine Schulen im alpinen Raum von Bedeutung sind. Zusammenfassend kann festgehalten werden, dass folgende allgemeine Faktoren in der Planung und Umsetzung eines Schulentwicklungsprojekts an kleinen Schulen zu beachten sind:

- Kommunikation: Eine gute Kommunikation zwischen den Akteuren und allen Beteiligten des Schulentwicklungsprozesses ist der wichtigste Faktor, der zu einer erfolgreichen Implementation einer Innovation führt.

- Zeitmanagement: In der Regel umfasst ein Schulentwicklungsprozess von der Absichtserklärung bis hin zur Implementation einige Jahre, meist sind fünf bis zehn Jahre erforderlich. Oft sollten Entwicklungsvorhaben an kleinen Schulen jedoch innert kurzer Zeit realisiert werden, was mitunter dazu führen kann, dass Schulentwicklungsprojekte ins Stocken geraten oder gar zum Scheitern verurteilt sind. Die Beteiligten benötigen ausreichend Zeit, um sich mit den geplanten Veränderungen auseinanderzusetzen.

- Einbezug aller Beteiligten: Der Einbezug aller Beteiligten, vor allem der Lehrpersonen, aber auch der Eltern und der Bevölkerung der Gemeinde, dient dazu, Widerständen zu begegnen und Vorbehalte auszuräumen. Wichtig ist vor allem, dass die Lehrpersonen als Team am Prozess teilhaben können, damit sie sich mit dem Projekt identifizieren und dieses als Team gemeinsam tragen.

- Arbeitsgruppe: Die Beteiligung verschiedener Akteure der Schule am Projekt beispielsweise in Form einer Arbeitsgruppe erweist sich als vorteilhaft, denn dadurch werden die verschiedenen Perspektiven ins Projekt eingebracht und die unterschiedlichen Bedürfnisse berücksichtigt.

- Begleitung durch Fachpersonen/Externe Beratung: Eine externe Begleitung bzw. Beratung des Entwicklungsprozesses ist empfehlenswert, denn die Aufgaben der Arbeitsgruppe sind äusserst komplex. Eine professionelle Projektbegleitung bringt Fähigkeiten und Fertigkeiten mit, über die Personen in der Arbeitsgruppe in der Regel nicht verfügen. 
- Fokussierung auf die Qualität des Unterrichts: Die Schule sollte sich durch Schülerorientierung auszeichnen und der Unterricht sollte das Kerngeschäft der Schule sein. Es besteht jedoch die Gefahr, dass infolge des Schulentwicklungsprozesses die Schülerinnen und Schüler sowie der Unterricht in den Hintergrund gedrängt werden. Dies ist zu vermeiden, denn das Wohl der Kinder muss immer, auch in und während Schulentwicklungsprozessen, im Zentrum stehen.

- Vorausplanung: Die Schule entwickelt sich, sowohl geplant als auch zufällig. Deshalb ist ein Entwicklungsprozess nicht einfach abgeschlossen. Es ist wichtig, künftige Entwicklungen zu planen, um sich auf anstehende Veränderungen entsprechend vorzubereiten.

- Evaluation: Die Bedeutung der Evaluation von Entwicklungsprojekten wird vielfach unterschätzt. Im Rahmen der Evaluation werden Ziele überprüft und neue gesetzt. Rolff (2001) meint dazu: «[N]ur eine Schule, die ihre Arbeitsgrundlage und ihre Lernergebnisse fortlaufend überprüft, die ihre Stärken und Schwächen analysiert und diagnostiziert, kann ihren Unterricht im Sinne einer lernenden Schule selber weiterentwickeln» (S. 102).

Dazu kommen spezifische Faktoren, die besonders in einem sich wandelnden ländlichen Raum ihren Einfluss haben:

- Das Zusammenspiel der Regional- und Schulentwicklung: Es zeigt sich, dass Schul- und Regionalentwicklung einen sehr wichtigen Beitrag für das Überleben einer Region und somit auch der Schulen darstellen. Schul- und Regionalentwicklung müssen Hand in Hand gehen, damit die Standortattraktivität der Schulen gewährleistet werden kann.

- Finanzielle Unterstützung: Für die Realisierung von Schulentwicklungsprojekten und der Implementation von Innovationen muss mit zusätzlichen Kosten gerechnet werden. Für kleine Gemeinden im alpinen Raum machen die Schulausgaben einen grossen Teil des Gesamtbudgets aus. Aus der Sicht der Akteure ist es wichtig, genügend Geld für die Schule zu budgetieren, damit Innovationen nicht Sparmassnahmen zum Opfer fallen. Die Schule soll gute Angebote für die Kinder bereitstellen können, weshalb Entwicklungsprojekte finanziell unterstützt werden sollten. Dazu braucht es jedoch auch eine finanzielle Unterstützung seitens des Kantons bzw. des Bundes, denn vor allem finanzschwache Gemeinden verfügen nicht immer über die dafür erforderlichen Mittel. Schulen, die ,etwas machen', wünschen sich Anerkennung, was für sie auch durch die finanzielle Unterstützung seitens der Gemeinde und/oder des Kantons zum Ausdruck gebracht werden kann.

- Erfahrungsaustausch/Netzwerke: Die kleinen Schulen wünschen sich ein Netzwerk kleiner Schulen, welche gleiche oder ähnliche Entwicklungen schon durchlaufen haben. Hospitationen an anderen kleinen und innovativen Schulen erachten die Akteure als hilfreich, damit Erfahrungen ausgetauscht 
werden können. Es kann auch als externe Beratung angesehen werden, wenn Vertreter anderer Schulen die Funktion ,kritischer Freunde' einnehmen und Rückmeldungen über Beobachtungen und Eindrücke geben.

- Ausbildung/Weiterbildung. Die Ausbildung und Weiterbildung der Lehrpersonen im Bereich des altersgemischten Unterrichtens (Unterrichten an kleinen Schulen) wird von den Akteuren vermehrt gewünscht, denn dies komme derzeit in der Ausbildung und in der Weiterbildung zu kurz.

\section{Fazit}

Im Rahmen dieses Beitrags wurden Fallbeispiele von innovativen kleinen Schulen aufgezeigt. Innovative Lösungsansätze zeichnen sich durch einen Einklang zwischen Bedürfnissen der Region und der Schulen aus, diese werden Hand in Hand geplant und implementiert. Es konnte gezeigt werden, dass die Rahmenbedingungen (vor allem die sinkenden Schülerzahlen) an den kleinen Schulen die Entwicklungen auslösen, diese Situation jedoch zusätzlich von innovativen Schulen zur Steigerung der Attraktivität sowie der pädagogischen Qualität genutzt werden. Den treibenden Kräften, die als ,Motor' von Schulentwicklungsprozessen angeschaut werden können, kommen im Prozess eine grosse Bedeutung zu. Dies sind meistens Schulratspräsidenten, Schulleiter oder Lehrpersonen. Ohne sie würden solche Prozesse oft nicht zu Ende geführt. Das hohe Mitspracherecht der Lehrpersonen an kleinen Schulen zeigt einen positiven Zusammenhang mit der Innovationsbereitschaft derselben Lehrpersonen. Je mehr Mitspracherecht die Lehrpersonen also haben, desto innovativer zeigen sie sich, und umgekehrt.

Es gibt Gemeinden, die für ihre Regionalentwicklung geographisch unvorteilhaft liegen, beispielsweise weit von den Zentren entfernt, hinter einem Pass oder in einem abgelegenen Tal. Für diese Regionen ist es sehr wichtig, innovativ zu bleiben und über zukünftige Möglichkeiten nachzudenken. Ausreichende Arbeitsplätze aus verschiedenen Berufsgruppen bilden eine wichtige Voraussetzung dafür, dass Familien mit Kindern in einer Gemeinde wohnhaft bleiben bzw. sich ansiedeln (vgl. Michelet in dieser Nummer). Die Schule spielt bei der Wahl des Wohnortes insofern eine zentrale Rolle, als ihr Angebot sowie ihr Ruf darüber entscheidet, ob Familien mit Kindern die Gemeinde als Wohnsitz wählen. Für die Schulen und deren Entwicklung ist die kantonale Strategie bzw. diejenige des Bundeslands im Umgang mit den Kleinschulen von grosser Bedeutung. In Vorarlberg wird bewusst darauf geachtet, dass jede Gemeinde mindestens eine Grundschule führen kann; die Kantone Graubünden, St. Gallen und Wallis versuchen eher, die Schulen zu zentralisieren. Solche Entscheidungen haben unterschiedliche Auswirkungen auf kleine Schulen im alpinen Raum und deren Entwicklung. Für die Umsetzung der Regional- sowie Schulentwicklung sind treibende Kräfte wichtig. Es braucht in den entsprechenden Gremien engagierte 
Menschen, die gewillt sind, die Schule sowie die Region weiterzuentwickeln. Leider zeigt sich immer wieder, dass die Dörfer in einem Tal untereinander oder innerhalb einer Region in Konkurrenz stehen, was sich hemmend auf die Entwicklungsprozesse auswirkt. Nur mit einer gemeinsamen Vision und einem gemeinsamen Willen lässt sich eine Region zukunftsfähig weiterentwickeln. Die regionale Zusammenarbeit ist eine sehr wichtige Komponente dafür, dass Synergien auch genutzt werden können.

\section{Anmerkungen}

1 In dieser Studie wird der alpine Raum exemplarisch für Verdichtungs- Entleerungsprozesse untersucht. Zum Begriff des ländlichen Raumes siehe die Einleitung von Périsset und Steiner in dieser Nummer.

2 In Vorarlberg gibt es keine Schulräte, dagegen in jeder Schule eine Schulleitung. Im Wallis und in Graubünden wird an allen Schulen lokale Schulbehörden eingesetzt, eine Schulleitung gibt es aber nicht in allen Schulen.

\section{Literaturverzeichnis}

Altrichter, H. \& Wiesinger, S. (2005). Implementation von Schulinnovationen - aktuelle Hoffnungen und Forschungswissen. Journal für Schulentwicklung, 9 ( 4), 28-36.

Bätzing, W. (2005). Die Alpen. Geschichte und Zukunft einer europäischen Kulturlandschaft. München: C.H. Beck

Bohl, T. (2009). Theorien und Konzepte der Schulentwicklung. In S. Blömeke, T. Bohl, L. Haag, G. Lang-Wojtasik \& W. Sacher (Hrsg.), Handbuch Schule (S. 553-559). Bad Heilbrunn: Verlag Julius Klinkhardt.

Dalin, P. (1999). Theorie und Praxis der Schulentwicklung. Neuwied, Kriftel: Luchterhand Verlag.

Dalin, P. \& Rolff, H.-G. (1997). Der institutionelle Schulentwicklungsprozess (ISP) (3. Auflage). Bönen: Klettler.

Fend, H. (1986). «Gute Schulen - schlechte Schulen». Die einzelne Schule als pädagogische Handlungseinheit. Die Deutsche Schule, 78 (3), 275-293.

Flick, U. (2000). Qualitative Forschung. Theorie, Methoden, Anwendung in Psychologie und Sozialwissenschaften. Reinbek bei Hamburg: Rowohlt.

Grunder, H.-U. (2002). Schulentwicklung durch Kooperation und Vernetzung. Schule verändern. Bad Heilbrunn: Klinkhardt Forschung.

Hunziker, M. (2007). Externe Evaluation von Kleinschulen. Bestandesaufnahme mit Lösungsmöglichkeiten. Fachstelle Externe Schulevaluation, Kanton Thurgau. Zugriff am 21.12.2012 unter http://www.argev.ch/page.php?pages_id=12\&language=de

Kerle, U. (2007). Perspektiven der Volksschule im Kanton Graubünden. Eine empirische Studie im Kontext aktueller demographischer und bildungspolitischer Veränderungen. Chur: Südostschweiz Buchverlag.

Mayring, P. (2008). Einführung in die qualitative Sozialforschung (4. Auflage). Weinheim: Beltz-Verlag.

Michelet, J., Steiner, E., Périsset, D., Eyer, Ph. \& Bumann, C. (2011). Sozio-ökonomische Veränderungen in Vorarlberg und in den Kantonen Graubünden, St. Gallen und Wallis. In R. Müller, A. Keller, U. Kerle, A. Raggl \& E. Steiner (Hrsg.), Schule im alpinen Raum (S. 17-73). Innsbruck: Studienverlag.

Müller, R., Keller, A., Kerle, U., Raggl, A. \& Steiner, E. (Hrsg.). (2011). Schule im alpinen Raum. Innsbruck: Studienverlag.

Rahm, S. (2005). Einführung in die Theorie der Schulentwicklung. Weinheim: Beltz Verlag. 
Rolff, H.-G. (2001). Schulentwicklung konkret: Steuergruppe-Bestandesaufnahme-Evaluation. Institut für schulische Fortbildung und schulpsychologische Beratung des Landes Rheinland-Pfalz (IFS). Seelze-Velber. Kallmeyer.

Rolff, H.-G. (2006). Unterrichtsentwicklung als Schulentwicklung. Jahrbuch der Schulentwicklung, 14, 22-245.

Rolff, H.-G. (2007). Studien zu einer Theorie der Schulentwicklung. Weinheim: Beltz Verlag.

Rolff, H.-G. (2010). Schulentwicklung als Trias von Organisations-, Unterrichts- und Personalentwicklung. In T. Bohl, W. Helsper, H. G. Holtappels \& C. Schelle (Hrsg.), Handbuch Schulentwicklung (S. 29-36). Bad Heilbrunn: Verlag Julius Klinkhardt.

Schaumburg, H., Prasse, D. \& Blömeke, S. (2010). Implementation von Innovationen in der Schule. In S. Blömeke, T. Bohl, L. Haag, G. Lang-Wojtasik \& W. Sacher (Hrsg.), Handbuch Schule (S. 596-600). Bad Heilbrunn: Verlag Julius Kinkhardt.

Schumacher, L. (2008). Wodurch wird die Bereitschaft von Lehrkräften zur Mitarbeit an Schulentwicklungsprojekten beeinflusst? In E.-M. Lankes (Hrsg.), Pädagogische Professionalität als Gegenstand empirischer Forschung. Münster: Waxmann.

Sutter, B. \& Kerle, U. (2011). Kleine Schulen im alpinen Raum suchen neue Wege. In R. Müller, A. Keller, U. Kerle, A. Raggl \& E. Steiner (Hrsg.), Schule im alpinen Raum (S. 307-376). Innsbruck: Studienverlag.

Warwas, J., Jürgen, S. \& Meier, M. (2008). Change Management von Schulen - Erfolgsfaktoren und Handlungsstrategien aus Sicht der Schulleitung an beruflichen Schulen. In R. Voss (Hrsg.), Innovatives Schulmanagement (S. 102-124). Gernsbach: Deutscher Betriebswirte-Verlag.

Schlagworte: Schulentwicklung, Implementation von Innovationen, Change Management, Kleine Schulen

Schlagworte: Schulentwicklung, Implementation von Innovationen, Change Management, Kleine Schulen

\section{Le développement des «petites écoles» dans les régions rurales}

\section{Ré sumé}

Cet article traite du développement des petites écoles dans quelques régions rurales de trois régions linguistiques des Alpes. Il y est présenté certaines innovations proposées par de petites écoles au niveau local. La question de l'identification des facteurs qui animent le développement des petites écoles est également débattue. En outre, cet article vise à mettre en évidence certains facteurs du succès de l'implémentation durable des innovations, facteurs qui semblent particulièrement permettre le maintien des "petites écoles» dans les régions rurales.

Mots-clés: Développement des écoles, implémentation des innovations, management du changement, petites écoles 


\section{Sviluppo scolastico di piccole scuole in regioni rurali}

Riassunto

La presente relazione si occupa dei processi di sviluppo di piccole scuole in regioni rurali dello spazio alpino di tre regioni linguistiche. Basandosi su vari casi esemplari attuali, vengono discusse possibili soluzioni e innovazioni a favore delle piccole scuole sia a livello locale che regionale. Si cerca di dare una risposta alla domanda: Quali fattori specifici favoriscono processi di sviluppo scolastico nelle piccole scuole? Infine l'articolo mette in evidenza fattori favorevoli per l'implementazione durevole di innovazioni per il mantenimento di piccole scuole nelle regioni rurali.

Parole chiave: Sviluppo scolastico, implementazione di innovazioni, change management, piccole scuole

\section{Development of "small schools" in rural areas}

\section{Summary}

This article deals with the development of small schools in rural alpine areas in three different linguistic regions. On the basis of case studies, a number of small schools' different local inventive solutions will be discussed. The question of which influencing variables drive innovative developments will also be analysed. Additionally, this article aims to identify factors of success for the sustainable implementation of innovations to preserve small schools in peripheral regions.

Key words: Development of schools, implementation of innovations, change management, small schools 
